\title{
Millimeter-Wave Rotational Spectrum, Barrier to Internal Rotation, and DFT Calculation of $o$-Tolunitrile
}

\author{
A. I. Jaman, ${ }^{1}$ P. Hemant Kumar, ${ }^{2}$ and P. R. Bangal ${ }^{2}$ \\ ${ }^{1}$ Experimental Condensed Matter Physics Division, Saha Institute of Nuclear Physics, Sector-1, Block-AF, Bidhannagar, \\ Kolkata 700 064, India \\ ${ }^{2}$ Inorganic and Physical Chemistry Division, Indian Institute of Chemical Technology, Uppal Road, Tarnaka, \\ Hyderabad 500 607, India
}

Correspondence should be addressed to A. I. Jaman, aismail.jaman@saha.ac.in and P. R. Bangal, prakriti@iict.res.in

Received 21 March 2011; Revised 15 June 2011; Accepted 16 June 2011

Academic Editor: Emil Proynov

Copyright ( $) 2011$ A. I. Jaman et al. This is an open access article distributed under the Creative Commons Attribution License, which permits unrestricted use, distribution, and reproduction in any medium, provided the original work is properly cited.

The millimeter-wave rotational spectra of $o$-tolunitrile $\left(\mathrm{C}_{6} \mathrm{H}_{4} \mathrm{CH}_{3} \mathrm{CN}\right)$ have been investigated in the ground torsional state in the frequency range 50.0-75.0 GHz. Many high-J rotational lines with large A-E splitting due to internal rotation of the methyl top have been assigned. A least squares analysis of the A-E splitting of 92 transitions resulted in the determination of accurate values of internal rotation parameters. The observed parameters were compared with the previously reported experimental values and DFT calculation results.

\section{Introduction}

The microwave rotational spectra of molecules having a methyl top attached to a planar frame shows a characteristic splitting corresponding to A and E symmetry of the torsional states. These A-E doublets may be closely or widely spaced depending on the height of the potential barrier and transitions studied. Hence, an analysis of the observed A-E splitting would lead to the determination of the barrier height hindering the internal rotation. Microwave spectroscopic techniques have been widely employed to study the effect of internal rotation of the methyl group on the rotational spectra of the molecules concerned.

Extensive studies on the microwave [1-5] and electronic [6-8] spectra of toluene and substituted toluenes have been reported in the literature. In contrast, not much microwave spectroscopic work has been reported on tolunitriles. Fujii et al. [9] have investigated the fluorescence excitation and dispersed fluorescence spectra of $o-, m$-, and $p$-tolunitrile in supersonic jets. By analyzing the low-frequency bands due to internal rotation of the methyl group they have successfully determined the height of the potential barrier hindering the internal rotation for $m$ - and $p$-tolunitrile. However, in the case of $o$-tolunitrile, the absence of low-frequency bands prevented them from determining the potential barrier.
In an earlier communication, Jaman et al. [10] have reported the rotational constants and a value of the potential barrier $V_{3}$ from an analysis of the A-E splitting of the microwave spectrum of $o$-tolunitrile in the ground torsional state. Later, Hansen et al [11] have investigated the high-resolution microwave spectrum of $o$-tolunitrile by using molecular beam FT microwave spectroscopic technique and determined rotational, centrifugal distortion constants, ${ }^{14} \mathrm{~N} \mathrm{nu}-$ clear quadrupole hyperfine constants, as well as $V_{3}$. The internal rotation parameters reported in $[10,11]$ were in good agreement with each other.

In the present communication, we have extended the analysis of the microwave spectra of $o$-tolunitrile to high- $J$ rotational transitions where the A-E splittings are large. The inclusion of high- $J$ rotational transitions in the least-squares analysis has improved the accuracy of the molecular constants. Furthermore, a detailed DFT calculation on the molecular structure, molecular constants, and internal rotation parameters are reported and compared with the experimental values.

\section{Experimental Details}

The sample of $o$-tolunitrile was obtained from Aldrich Chemical Co. (quoted purity: 98\%) and was used without 
TABLE 1: Observed rotational transition frequencies $(\mathrm{MHz})$ of the A-species of $o$-tolunitrile in the ground torsional state.

\begin{tabular}{|c|c|c|c|c|c|c|c|c|}
\hline \multicolumn{7}{|c|}{ Transition } & \multirow[t]{2}{*}{ Frequency } & \multirow[t]{2}{*}{ Obs.-Cal. } \\
\hline$J^{\prime}$ & $K_{-1}^{\prime}$ & $K_{+1}^{\prime}$ & $\leftarrow$ & $J$ & $K_{-1}$ & $K_{+1}$ & & \\
\hline 9 & 7 & 3 & & 8 & 7 & 2 & $22919.90^{\mathrm{a}}$ & -.36 \\
\hline 10 & 2 & 9 & & 9 & 2 & 8 & $22802.20^{\mathrm{a}}$ & -.13 \\
\hline 10 & 4 & 7 & & 9 & 4 & 6 & $25605.20^{\mathrm{a}}$ & -.34 \\
\hline 10 & 6 & 5 & & 9 & 6 & 4 & $25692.90^{\mathrm{a}}$ & -.16 \\
\hline 10 & 6 & 4 & & 9 & 6 & 3 & $25720.60^{\mathrm{a}}$ & .04 \\
\hline 10 & 7 & 3 & & 9 & 7 & 2 & $25546.60^{\mathrm{a}}$ & -.44 \\
\hline 10 & 8 & 2 & & 9 & 8 & 1 & $25446.90^{\mathrm{a}}$ & .02 \\
\hline 10 & 9 & 1 & & 9 & 9 & 0 & $25380.00^{\mathrm{a}}$ & .01 \\
\hline 13 & 5 & 9 & & 12 & 5 & 8 & $33567.45^{\mathrm{a}}$ & -.20 \\
\hline 13 & 6 & 8 & & 12 & 6 & 7 & $33776.80^{\mathrm{a}}$ & .00 \\
\hline 13 & 6 & 7 & & 12 & 6 & 6 & $34250.80^{\mathrm{a}}$ & -.11 \\
\hline 13 & 7 & 7 & & 12 & 7 & 6 & $33579.65^{\mathrm{a}}$ & -.10 \\
\hline 13 & 7 & 6 & & 12 & 7 & 5 & $33628.74^{\mathrm{a}}$ & .12 \\
\hline 13 & 9 & 5 & & 12 & 9 & 4 & $33217.40^{\mathrm{a}}$ & .28 \\
\hline 14 & 4 & 11 & & 13 & 4 & 10 & $34626.90^{\mathrm{a}}$ & -.20 \\
\hline 14 & 7 & 8 & & 13 & 7 & 7 & $36305.60^{\mathrm{a}}$ & -.25 \\
\hline 14 & 7 & 7 & & 13 & 7 & 6 & $36433.93^{\mathrm{a}}$ & .15 \\
\hline 14 & 8 & 7 & & 13 & 8 & 6 & $36058.00^{\mathrm{a}}$ & .32 \\
\hline 14 & 8 & 6 & & 13 & 8 & 5 & $36067.50^{\mathrm{a}}$ & .15 \\
\hline 15 & 2 & 13 & & 14 & 2 & 12 & $34764.70^{\mathrm{a}}$ & .09 \\
\hline 15 & 3 & 12 & & 14 & 3 & 11 & $36977.20^{\mathrm{a}}$ & -.14 \\
\hline 15 & 4 & 12 & & 14 & 4 & 11 & $36690.20^{\mathrm{a}}$ & .14 \\
\hline 15 & 6 & 10 & & 14 & 6 & 9 & $39049.10^{\mathrm{a}}$ & -.06 \\
\hline 15 & 7 & 9 & & 14 & 7 & 8 & $39041.60^{\mathrm{a}}$ & .24 \\
\hline 15 & 7 & 8 & & 14 & 7 & 7 & $39339.60^{\mathrm{a}}$ & -.27 \\
\hline 15 & 8 & 8 & & 14 & 8 & 7 & $38774.90^{\mathrm{a}}$ & .26 \\
\hline 15 & 8 & 7 & & 14 & 8 & 6 & $38803.40^{\mathrm{a}}$ & .27 \\
\hline 16 & 4 & 13 & & 15 & 4 & 12 & $38713.60^{\mathrm{a}}$ & .35 \\
\hline 18 & 0 & 18 & & 17 & 0 & 17 & $36750.20^{\mathrm{a}}$ & .07 \\
\hline 20 & 7 & 13 & & 19 & 7 & 12 & 55324.10 & .17 \\
\hline 21 & 7 & 14 & & 20 & 7 & 13 & 58107.00 & -.08 \\
\hline 21 & 8 & 14 & & 20 & 8 & 13 & 55125.30 & .05 \\
\hline 21 & 9 & 12 & & 20 & 9 & 11 & 55578.20 & .30 \\
\hline 21 & 9 & 13 & & 20 & 9 & 12 & 55076.80 & .24 \\
\hline 22 & 6 & 16 & & 21 & 6 & 15 & 57746.00 & -.30 \\
\hline 22 & 7 & 15 & & 21 & 7 & 14 & 60513.00 & .01 \\
\hline 22 & 8 & 14 & & 21 & 8 & 13 & 60609.60 & -.16 \\
\hline 22 & 9 & 13 & & 21 & 9 & 12 & 58791.90 & -.11 \\
\hline 22 & 12 & 10 & & 21 & 12 & 9 & 56794.00 & -.21 \\
\hline 22 & 13 & 9 & & 21 & 13 & 8 & 56556.80 & -.31 \\
\hline 22 & 14 & 8 & & 21 & 14 & 7 & 56374.80 & -.01 \\
\hline 22 & 15 & 7 & & 21 & 15 & 6 & 56230.60 & .20 \\
\hline 22 & 16 & 6 & & 21 & 16 & 5 & 56113.80 & .28 \\
\hline 22 & 17 & 5 & & 21 & 17 & 4 & 56017.10 & -.20 \\
\hline 22 & 18 & 4 & & 21 & 18 & 3 & 55937.00 & .03 \\
\hline 22 & 19 & 3 & & 21 & 19 & 2 & 55868.80 & -.28 \\
\hline 22 & 20 & 2 & & 21 & 20 & 1 & 55811.10 & -.01 \\
\hline 23 & 21 & 2 & & 22 & 21 & 1 & 58342.10 & .17 \\
\hline 23 & 20 & 3 & & 22 & 20 & 2 & 58399.20 & .19 \\
\hline 23 & 19 & 4 & & 22 & 19 & 3 & 58465.50 & .21 \\
\hline 23 & 18 & 5 & & 22 & 18 & 4 & 58543.00 & .02 \\
\hline 23 & 17 & 6 & & 22 & 17 & 5 & 58634.90 & -.13 \\
\hline 23 & 16 & 7 & & 22 & 16 & 6 & 58745.40 & -.04 \\
\hline
\end{tabular}


TABle 1: Continued.

\begin{tabular}{|c|c|c|c|c|c|c|c|c|}
\hline & \multicolumn{5}{|c|}{ Transition } & \multirow{2}{*}{\multicolumn{2}{|c|}{ Frequency }} & \multirow[t]{2}{*}{ Obs.-Cal. } \\
\hline$J^{\prime}$ & $K_{-1}^{\prime}$ & $K_{+1}^{\prime}$ & $\leftarrow$ & $J$ & $K_{-1}$ & & & \\
\hline 23 & 15 & 8 & & 22 & 15 & 7 & 58880.00 & .14 \\
\hline 23 & 14 & 9 & & 22 & 14 & 8 & 59046.40 & -.06 \\
\hline 23 & 13 & 10 & & 22 & 13 & 9 & 59257.70 & -.10 \\
\hline 23 & 12 & 11 & & 22 & 12 & 10 & 59535.40 & -.14 \\
\hline 23 & 11 & 12 & & 22 & 11 & 11 & 59931.00 & .07 \\
\hline 23 & 10 & 13 & & 22 & 10 & 12 & 60633.20 & -.13 \\
\hline 23 & 9 & 14 & & 22 & 9 & 13 & 62173.00 & .17 \\
\hline 23 & 6 & 17 & & 22 & 6 & 16 & 59400.50 & .22 \\
\hline 24 & 23 & 1 & & 23 & 23 & 0 & 60823.00 & -.11 \\
\hline 24 & 22 & 2 & & 23 & 22 & 1 & 60872.60 & -.05 \\
\hline 24 & 21 & 3 & & 23 & 21 & 2 & 60929.10 & .14 \\
\hline 24 & 20 & 4 & & 23 & 20 & 3 & 60993.80 & -.03 \\
\hline 24 & 19 & 5 & & 23 & 19 & 4 & 61068.90 & -.33 \\
\hline 24 & 18 & 6 & & 23 & 18 & 5 & 61157.60 & -.09 \\
\hline 24 & 17 & 7 & & 23 & 17 & 6 & 61262.60 & -.05 \\
\hline 24 & 16 & 8 & & 23 & 16 & 7 & 61388.80 & .01 \\
\hline 24 & 15 & 9 & & 23 & 15 & 8 & 61542.60 & -.12 \\
\hline 24 & 14 & 10 & & 23 & 14 & 9 & 61734.20 & -.01 \\
\hline 24 & 13 & 11 & & 23 & 13 & 10 & 61978.30 & -.35 \\
\hline 24 & 12 & 12 & & 23 & 12 & 11 & 62305.00 & .19 \\
\hline 24 & 12 & 13 & & 23 & 12 & 12 & 62297.00 & .07 \\
\hline 24 & 11 & 13 & & 23 & 11 & 12 & 62793.30 & -.22 \\
\hline 24 & 11 & 14 & & 23 & 11 & 13 & 62709.60 & .19 \\
\hline 24 & 6 & 18 & & 23 & 6 & 17 & 61127.50 & .12 \\
\hline 25 & 6 & 19 & & 24 & 6 & 18 & 62935.70 & .06 \\
\hline 25 & 8 & 17 & & 24 & 8 & 16 & 68834.00 & -.07 \\
\hline 25 & 9 & 16 & & 24 & 9 & 15 & 69015.60 & .09 \\
\hline 25 & 9 & 17 & & 24 & 9 & 16 & 65732.10 & .28 \\
\hline 25 & 10 & 15 & & 24 & 10 & 14 & 67048.00 & .09 \\
\hline 25 & 24 & 1 & & 24 & 24 & 0 & 63354.70 & .38 \\
\hline 25 & 23 & 2 & & 24 & 23 & 1 & 63403.20 & -.07 \\
\hline 25 & 22 & 3 & & 24 & 22 & 2 & 63458.70 & -.18 \\
\hline 26 & 8 & 18 & & 25 & 8 & 17 & 70796.70 & .10 \\
\hline 26 & 10 & 16 & & 25 & 10 & 15 & 70507.00 & .18 \\
\hline 26 & 12 & 14 & & 25 & 12 & 13 & 67953.00 & -.16 \\
\hline 26 & 14 & 12 & & 25 & 14 & 11 & 67163.60 & .06 \\
\hline 26 & 22 & 4 & & 25 & 22 & 3 & 66051.30 & -.01 \\
\hline 27 & 9 & 19 & & 26 & 9 & 18 & 70443.10 & .05 \\
\hline 27 & 11 & 17 & & 26 & 11 & 16 & 71220.20 & -.27 \\
\hline 27 & 12 & 15 & & 26 & 12 & 14 & 70856.10 & .06 \\
\hline 27 & 13 & 14 & & 26 & 13 & 13 & 70288.20 & -.21 \\
\hline 28 & 22 & 6 & & 27 & 22 & 5 & 71256.00 & .13 \\
\hline 28 & 24 & 4 & & 27 & 24 & 3 & 71108.90 & -.10 \\
\hline 28 & 26 & 2 & & 27 & 26 & 1 & 70994.40 & .11 \\
\hline 29 & 22 & 7 & & 28 & 22 & 6 & 73868.50 & -.10 \\
\hline 29 & 24 & 5 & & 28 & 24 & 4 & 73705.30 & .03 \\
\hline 29 & 25 & 4 & & 28 & 25 & 3 & 73637.80 & -.03 \\
\hline 29 & 26 & 3 & & 28 & 26 & 2 & 73577.80 & -.09 \\
\hline 29 & 27 & 2 & & 28 & 27 & 1 & 73524.40 & .09 \\
\hline 29 & 28 & 1 & & 28 & 28 & 0 & 73476.20 & .03 \\
\hline 30 & 7 & 24 & & 29 & 7 & 23 & 72553.20 & -.02 \\
\hline
\end{tabular}


TABLE 2: Observed effective rotational, centrifugal distortion constants and inertia defect of the A-species of $o$-tolunitrile in the ground torsion state.

\begin{tabular}{lc}
\hline Constants & This work \\
\hline$A / \mathrm{MHz}$ & $2892.79(6)$ \\
$B / \mathrm{MHz}$ & 1500.409 \\
$C / \mathrm{MHz}$ & $(7)$ \\
$\triangle_{J} / \mathrm{kHz}$ & $993.507(8)$ \\
$\triangle_{J} / \mathrm{kHz}$ & $-0.032(7)$ \\
$\triangle_{K} / \mathrm{kHz}$ & $0.830(38)$ \\
$\delta_{j} / \mathrm{kHz}$ & $-1.31(41)$ \\
$\delta_{k} / \mathrm{kHz}$ & $0.045(4)$ \\
$\triangle=\left(I_{c}-I_{b}-I_{a}\right) / \mathrm{u} \AA^{2}$ & $-0.58(8)$ \\
& -2.85 \\
\hline
\end{tabular}

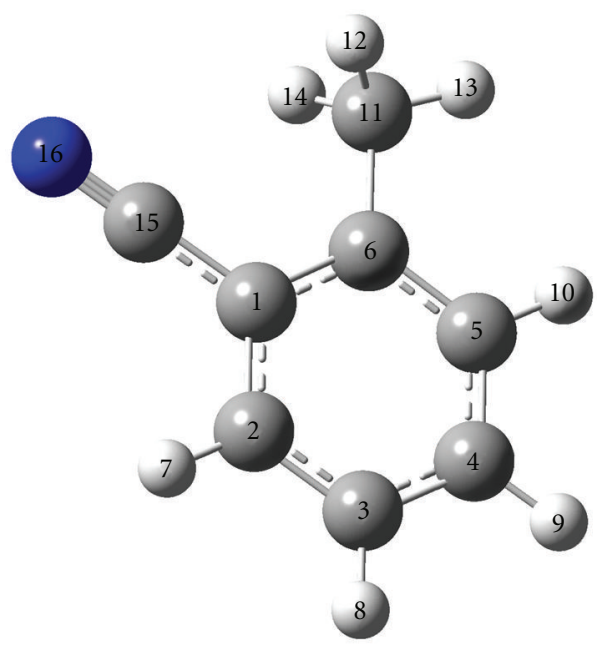

FIGURE 1: Molecular geometry of $o$-tolunitrile.

further purification. The millimeterwave spectrum was observed and measured with a conventional $100 \mathrm{kHz}$ Starkmodulated spectrometer in the frequency range 50.075.0 GHz. Okaya 24V10A and 35V10 klystrons followed by a frequency doubler (Millitech model MUD-15-H23FO) were used as frequency sources. The klystron frequency was swept by applying a sawtooth voltage on its repeller. The absorption line was modulated with a $100 \mathrm{kHz}$ square wave and phase sensitively detected by using a lock-in amplifier (EG \& G Princeton Applied Research, Model 124A). Absorption frequencies were measured at room temperature using a beat frequency technique where a frequency synthesizer (300.03000.0 MHz, Model BSVB13, Bonn Hungary Electronics) was used as a local oscillator. The sample pressure was maintained around 20-30 mTorr during the experiment. The method of frequency measurement was described in [5]. The Stark voltage was kept around $500 \mathrm{~V} / \mathrm{cm}$. The uncertainty in frequency measurement was estimated to be $\pm 0.15 \mathrm{MHz}$.

\section{Spectral Analysis}

Since the rotational constants and the potential barrier $V_{3}$ hindering the internal rotation are fairly well known from the

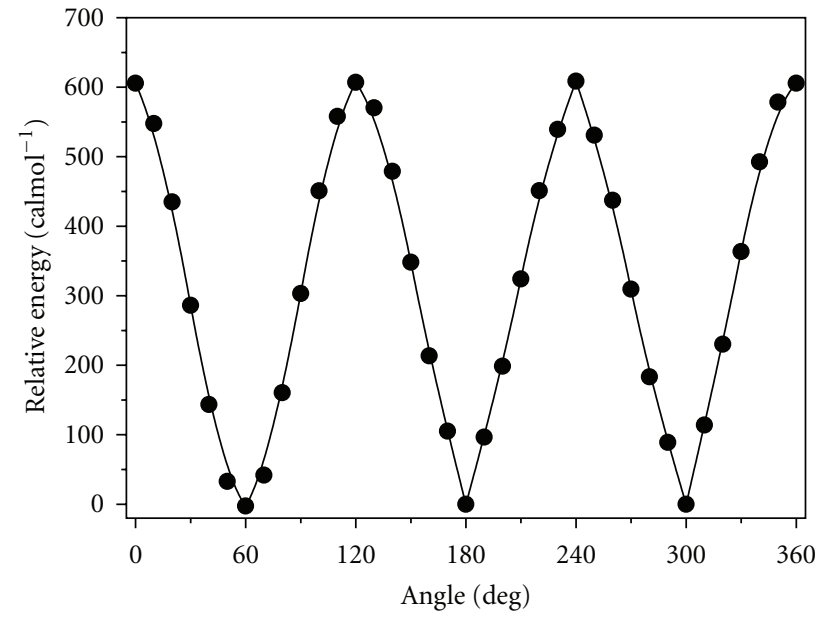

Figure 2: Potential function of the methyl torsion of $o$-tolunitrile as obtained from DFT calculation.

previous studies $[10,11]$ the assignments of the spectra for the high- $J$ transitions were completed without much difficulty. Initially, the A and E components of high- $J$ transitions were predicted in the frequency range $50.0-75.0 \mathrm{GHz}$ using the known values of the rotational constants and the potential barrier. The computer program KC3IAM assembled by Kasten [12] from a modified version [13] of Wood's classic treatment of a single top by the internal axis method [14] was used for prediction. Approximate values of A-E splitting were obtained for many transitions in the above frequency range. Around 100 transitions due to high $K_{-1}, K_{+1}$ with A-E splitting ranging from $6.3 \mathrm{MHz}$ to $178.4 \mathrm{MHz}$ were observed and measured.

Since the rotational Hamiltonian for the A-species transitions was similar in form to a rigid-rotor Hamiltonian with modified rotational constants [10] known as effective rotational constants [19] the A-species transitions were analyzed using the semirigid rotor Watson's A-reduction Hamiltonian ( $I^{\mathrm{r}}$-representation) [20]. Newly measured A-species transitions are listed in Table 1 along with the transitions reported earlier in [10]. In all, 104 transitions were used in the leastsquares analysis. Table 2 represents the values of effective rotational and centrifugal distortion constants and the value of the inertia defect for the A-species transitions after the final cycle of the fit. The standard deviation of the overall fit comes out to be $0.18 \mathrm{MHz}$.

The assigned E-species lines appear close to their predicted values. Table 3 shows the observed and measured Especies lines and the splitting due to internal rotation of the $\mathrm{CH}_{3}$ top. The observed A-E splittings of Table 3 were analyzed using KC3IAM program. An iterative least-squares analysis was performed with 92 observed A-E splitting which includes 12 from [10]. Two separate fits were performed. In Fit 1, three independent parameters, namely, the top moment of inertia $I_{\alpha}$, the potential barrier $V_{3}$ and $\theta_{a}$, the angle between the internal rotation axis and the principal axis $a$ were allowed to vary. In Fit 2, two independent parameters, 
TABLE 3: Observed rotational transition frequencies $(\mathrm{MHz})$ of the E-species of $o$-tolunitrile in the ground torsional state.

\begin{tabular}{|c|c|c|c|c|c|c|c|c|c|}
\hline$J^{\prime}$ & $K_{-1}^{\prime}$ & $K_{+1}^{\prime}$ & $\leftarrow$ & $J$ & $K_{-1}$ & $K_{+1}$ & Frequency & $\nu_{\mathrm{A}}-\nu_{\mathrm{E}}$ & Obs.-Calc. \\
\hline 13 & 7 & 6 & & 12 & 7 & 5 & $33584.23^{\mathrm{b}}$ & 44.51 & -0.15 \\
\hline 13 & 9 & 5 & & 12 & 9 & 4 & $33209.55^{\mathrm{b}}$ & 7.85 & 0.44 \\
\hline 14 & 4 & 11 & & 13 & 4 & 10 & $34619.10^{\mathrm{b}}$ & 7.80 & 0.09 \\
\hline 14 & 7 & 8 & & 13 & 7 & 7 & $36358.30^{\mathrm{b}}$ & -52.7 & -0.35 \\
\hline 14 & 7 & 7 & & 13 & 7 & 6 & $36352.38^{\mathrm{b}}$ & 81.55 & 0.42 \\
\hline 14 & 8 & 6 & & 13 & 8 & 5 & $36042.13^{\mathrm{b}}$ & 25.37 & -0.04 \\
\hline 15 & 3 & 13 & & 14 & 3 & 12 & $34735.40^{\mathrm{b}}$ & 6.30 & 0.47 \\
\hline 15 & 6 & 10 & & 14 & 6 & 9 & $39083.80^{\mathrm{b}}$ & -34.70 & -0.43 \\
\hline 15 & 7 & 8 & & 14 & 7 & 7 & $39207.80^{\mathrm{b}}$ & 131.80 & -0.16 \\
\hline 15 & 8 & 8 & & 14 & 8 & 7 & $38782.25^{\mathrm{b}}$ & -7.35 & 0.46 \\
\hline 15 & 8 & 7 & & 14 & 8 & 6 & $38765.50^{\mathrm{b}}$ & 37.90 & 0.31 \\
\hline 16 & 4 & 13 & & 15 & 4 & 12 & $38705.90^{\mathrm{b}}$ & 7.70 & -0.42 \\
\hline 21 & 7 & 14 & & 20 & 7 & 13 & 58077.80 & 29.20 & 0.48 \\
\hline 21 & 8 & 13 & & 20 & 8 & 12 & 57193.00 & 74.00 & 0.47 \\
\hline 21 & 8 & 14 & & 20 & 8 & 13 & 55149.10 & -23.80 & -0.27 \\
\hline 21 & 9 & 12 & & 20 & 9 & 11 & 55399.80 & 178.40 & 0.47 \\
\hline 22 & 9 & 13 & & 21 & 9 & 12 & 58624.00 & 167.60 & -0.25 \\
\hline 22 & 6 & 16 & & 21 & 6 & 15 & 57732.80 & 13.00 & -0.46 \\
\hline 22 & 7 & 15 & & 21 & 7 & 14 & 60489.60 & 23.40 & -0.26 \\
\hline 22 & 8 & 14 & & 21 & 8 & 13 & 60558.40 & 51.20 & 0.24 \\
\hline 22 & 12 & 10 & & 21 & 12 & 9 & 56763.20 & 30.80 & -0.26 \\
\hline 22 & 13 & 9 & & 21 & 13 & 8 & 56528.20 & 28.50 & -0.06 \\
\hline 22 & 14 & 8 & & 21 & 14 & 7 & 56347.80 & 27.00 & -0.14 \\
\hline 22 & 15 & 7 & & 21 & 15 & 6 & 56204.20 & 26.40 & 0.25 \\
\hline 22 & 16 & 6 & & 21 & 16 & 5 & 56088.20 & 25.80 & 0.40 \\
\hline 22 & 17 & 5 & & 21 & 17 & 4 & 55992.40 & 24.40 & -0.41 \\
\hline 22 & 18 & 4 & & 21 & 18 & 3 & 55912.30 & 24.70 & 0.33 \\
\hline 22 & 19 & 3 & & 21 & 19 & 2 & 55845.10 & 23.70 & -0.30 \\
\hline 23 & 6 & 17 & & 22 & 6 & 16 & 59387.40 & 13.10 & -0.15 \\
\hline 23 & 9 & 14 & & 22 & 9 & 13 & 62056.80 & 116.20 & -0.05 \\
\hline 23 & 10 & 13 & & 22 & 10 & 12 & 60476.00 & 157.20 & -0.23 \\
\hline 23 & 11 & 12 & & 22 & 11 & 11 & 59876.40 & 54.60 & 0.11 \\
\hline 23 & 12 & 11 & & 22 & 12 & 10 & 59501.20 & 34.20 & -0.38 \\
\hline 23 & 13 & 10 & & 22 & 13 & 9 & 59226.90 & 30.80 & 0.02 \\
\hline 23 & 14 & 9 & & 22 & 14 & 8 & 59017.00 & 29.40 & 0.36 \\
\hline 23 & 15 & 8 & & 22 & 15 & 7 & 58851.80 & 28.20 & 0.34 \\
\hline 23 & 16 & 7 & & 22 & 16 & 6 & 58718.30 & 27.10 & 0.12 \\
\hline 23 & 17 & 6 & & 22 & 17 & 5 & 58608.40 & 26.30 & 0.01 \\
\hline 23 & 18 & 5 & & 22 & 18 & 4 & 58517.10 & 25.90 & 0.12 \\
\hline 23 & 19 & 4 & & 22 & 19 & 3 & 58439.90 & 25.90 & 0.54 \\
\hline 23 & 20 & 3 & & 22 & 20 & 2 & 58374.00 & 25.40 & 0.39 \\
\hline 23 & 21 & 2 & & 22 & 21 & 1 & 58317.10 & 25.00 & 0.28 \\
\hline 24 & 6 & 18 & & 23 & 6 & 17 & 61113.90 & 13.60 & 0.02 \\
\hline 24 & 11 & 13 & & 23 & 11 & 12 & 62712.80 & 80.20 & -0.27 \\
\hline 24 & 11 & 14 & & 23 & 11 & 13 & 62740.00 & -30.00 & -0.11 \\
\hline 24 & 12 & 12 & & 23 & 12 & 11 & 62264.90 & 40.10 & 0.20 \\
\hline 24 & 13 & 11 & & 23 & 13 & 10 & 61945.10 & 33.20 & -0.07 \\
\hline 24 & 13 & 12 & & 23 & 13 & 11 & 61965.60 & 12.80 & 0.17 \\
\hline 24 & 14 & 10 & & 23 & 14 & 9 & 61702.80 & 31.40 & 0.35 \\
\hline 24 & 15 & 9 & & 23 & 15 & 8 & 61513.20 & 29.40 & -0.24 \\
\hline 24 & 15 & 10 & & 23 & 15 & 9 & 61529.00 & 13.60 & 0.09 \\
\hline 24 & 16 & 8 & & 23 & 16 & 7 & 61360.40 & 28.40 & -0.22 \\
\hline 24 & 16 & 9 & & 23 & 16 & 8 & 61375.30 & 13.50 & 0.03 \\
\hline 24 & 17 & 7 & & 23 & 17 & 6 & 61234.60 & 28.00 & 0.16 \\
\hline 24 & 17 & 8 & & 23 & 17 & 7 & 61249.30 & 13.30 & 0.01 \\
\hline 24 & 18 & 6 & & 23 & 18 & 5 & 61130.40 & 27.20 & -0.03 \\
\hline
\end{tabular}


TABle 3: Continued.

\begin{tabular}{|c|c|c|c|c|c|c|c|c|c|}
\hline$J^{\prime}$ & $\overline{K_{-1}^{\prime}}$ & $K_{+1}^{\prime}$ & $\leftarrow$ & $J$ & $K_{-1}$ & $K_{+1}$ & Frequency & $\nu_{\mathrm{A}}-\nu_{\mathrm{E}}$ & Obs.-Calc. \\
\hline 24 & 18 & 7 & & 23 & 18 & 6 & 61145.00 & 12.60 & -0.43 \\
\hline 24 & 19 & 5 & & 23 & 19 & 4 & 61042.30 & 26.60 & -0.14 \\
\hline 24 & 19 & 6 & & 23 & 19 & 5 & 61056.70 & 12.20 & -0.52 \\
\hline 24 & 20 & 4 & & 23 & 20 & 3 & 60967.30 & 26.50 & 0.16 \\
\hline 24 & 21 & 3 & & 23 & 21 & 2 & 60903.00 & 26.10 & 0.09 \\
\hline 24 & 22 & 2 & & 23 & 22 & 1 & 60847.00 & 25.60 & -0.11 \\
\hline 24 & 23 & 1 & & 23 & 23 & 0 & 60798.10 & 25.30 & -0.16 \\
\hline 25 & 24 & 1 & & 24 & 24 & 0 & 63328.00 & 26.70 & 0.25 \\
\hline 25 & 24 & 2 & & 24 & 24 & 1 & 63343.80 & 10.90 & -0.19 \\
\hline 25 & 23 & 2 & & 24 & 23 & 1 & 63376.50 & 26.70 & -0.01 \\
\hline 25 & 23 & 3 & & 24 & 23 & 2 & 63392.10 & 11.10 & -0.47 \\
\hline 25 & 22 & 3 & & 24 & 22 & 2 & 63431.40 & 27.30 & 0.31 \\
\hline 25 & 22 & 4 & & 24 & 22 & 3 & 63447.10 & 11.60 & -0.43 \\
\hline 25 & 6 & 19 & & 24 & 6 & 18 & 62921.80 & 14.10 & 0.12 \\
\hline 25 & 8 & 17 & & 24 & 8 & 16 & 68807.80 & 26.20 & 0.26 \\
\hline 25 & 9 & 17 & & 24 & 9 & 16 & 65728.90 & 3.20 & -0.11 \\
\hline 25 & 10 & 15 & & 24 & 10 & 14 & 66877.50 & 170.50 & -0.29 \\
\hline 25 & 22 & 4 & & 25 & 22 & 3 & 66022.80 & 28.50 & 0.21 \\
\hline 26 & 14 & 12 & & 25 & 14 & 11 & 67128.20 & 35.40 & -0.16 \\
\hline 26 & 12 & 14 & & 25 & 12 & 13 & 67887.10 & 65.90 & -0.22 \\
\hline 26 & 10 & 16 & & 25 & 10 & 15 & 70390.50 & 116.50 & -0.22 \\
\hline 26 & 8 & 18 & & 25 & 8 & 17 & 70775.70 & 21.00 & -0.09 \\
\hline 27 & 12 & 15 & & 26 & 12 & 14 & 70759.10 & 97.00 & -0.31 \\
\hline 27 & 9 & 19 & & 26 & 9 & 18 & 70426.50 & 17.00 & -0.10 \\
\hline 27 & 13 & 14 & & 26 & 13 & 13 & 70241.90 & 46.30 & -0.50 \\
\hline 27 & 11 & 17 & & 26 & 11 & 16 & 71365.20 & 145.00 & 0.09 \\
\hline 28 & 22 & 6 & & 27 & 22 & 5 & 71224.90 & 31.10 & 0.12 \\
\hline 28 & 24 & 4 & & 27 & 24 & 3 & 71078.90 & 30.00 & 0.21 \\
\hline 28 & 26 & 2 & & 27 & 26 & 1 & 70964.60 & 30.00 & 0.40 \\
\hline 29 & 22 & 7 & & 28 & 22 & 6 & 73836.10 & 32.40 & 0.04 \\
\hline 29 & 24 & 5 & & 28 & 24 & 4 & 73673.60 & 31.70 & 0.19 \\
\hline 29 & 25 & 4 & & 28 & 25 & 3 & 73606.70 & 31.10 & -0.05 \\
\hline 29 & 26 & 3 & & 28 & 26 & 2 & 73547.00 & 30.80 & -0.02 \\
\hline 29 & 27 & 2 & & 28 & 27 & 1 & 73493.90 & 30.50 & -0.02 \\
\hline 29 & 28 & 1 & & 28 & 28 & 0 & 73446.10 & 30.10 & -0.13 \\
\hline 30 & 7 & 24 & & 29 & 7 & 23 & 72544.30 & 8.90 & -0.25 \\
\hline
\end{tabular}

TABLE 4: Spectroscopic constants of $o$-tolunitrile from the fit of observed A-E splitting and DFT values.

\begin{tabular}{|c|c|c|c|}
\hline Constants & \multicolumn{2}{|c|}{ Experimental values } & DFT values \\
\hline$A / \mathrm{MHz}$ & \multicolumn{2}{|c|}{$2891.6469(5)^{\mathrm{c}}$} & 2884.36 \\
\hline$B / \mathrm{MHz}$ & \multicolumn{2}{|c|}{$1499.8809(1)^{\mathrm{c}}$} & 1506.90 \\
\hline $\mathrm{C} / \mathrm{MHz}$ & \multicolumn{2}{|c|}{$993.5217(6)^{\mathrm{c}}$} & 995.84 \\
\hline$\triangle_{J} / \mathrm{kHz}$ & \multicolumn{2}{|c|}{$0.039(1)^{\mathrm{c}}$} & 0.0403 \\
\hline$\triangle_{J K} / \mathrm{kHz}$ & \multicolumn{2}{|c|}{$0.396(5)^{c}$} & 0.327 \\
\hline$\triangle_{K} / \mathrm{kHz}$ & \multicolumn{2}{|c|}{$0.38(5)^{\mathrm{c}}$} & 0.601 \\
\hline$\delta_{j} / \mathrm{kHz}$ & \multicolumn{2}{|c|}{$0.0116(6)^{\mathrm{c}}$} & 0.0131 \\
\hline \multirow[t]{2}{*}{$\delta_{k} / \mathrm{kHz}$} & \multicolumn{2}{|c|}{$0.236(8)^{\mathrm{c}}$} & 0.206 \\
\hline & Fit 1 & Fit 2 & DFT values \\
\hline$I_{\alpha} / \mathrm{u} \AA^{2}$ & $3.23 \pm 0.01$ & 3.2 (fixed) & 3.25 \\
\hline$\theta_{a} /$ degree & $53.33 \pm 0.09$ & $53.50 \pm 0.03$ & 55.45 \\
\hline$\theta_{b} /$ degree & $36.67 \pm 0.09$ & $36.50 \pm 0.03$ & 34.55 \\
\hline$V_{3} / \mathrm{cal} / \mathrm{mol}$ & $533.47 \pm 2.8$ & $537.71 \pm 0.10$ & 608.41 \\
\hline
\end{tabular}

${ }^{c}[11]$ 
TABLE 5: Comparison of torsional barriers $\left(V_{3}\right)$ of toluene derivatives.

\begin{tabular}{lccc}
\hline Sample name & Chemical formula & $V_{3}(\mathrm{Cal} / \mathrm{mol})$ & Ref. \\
\hline$o$-Fluorotoluene & $o-\mathrm{C}_{6} \mathrm{H}_{4} \mathrm{CH}_{3} \mathrm{~F}$ & 649.30 & {$[2]$} \\
$o-{ }^{35}$ Chlorotoluene & $o-\mathrm{C}_{6} \mathrm{H}_{4} \mathrm{CH}_{3}{ }^{35} \mathrm{Cl}$ & $1328.524 / 1343.262^{\mathrm{d}}$ & {$[15,16]$} \\
$o-{ }^{37}$ Chlorotoluene & $o-\mathrm{C}_{6} \mathrm{H}_{4} \mathrm{CH}_{3}{ }^{37} \mathrm{Cl}$ & $1327.857 / 1339.976^{\mathrm{d}}$ & {$[15,16]$} \\
2,4-Difluorotoluene & $\mathrm{C}_{6} \mathrm{H}_{3} \mathrm{CH}_{3} \mathrm{~F}_{2}$ & $583.002 \pm 0.668$ & {$[5]$} \\
$o$-Xylene & $\mathrm{C}_{6} \mathrm{H}_{4} \mathrm{CH}_{3} \mathrm{CH}_{3}$ & $1482 \pm 9$ & $1883.809^{\mathrm{d}}$ \\
syn-o-Cresol & $s y n-o-\mathrm{C}_{6} \mathrm{H}_{4} \mathrm{CH}_{3} \mathrm{OH}$ & $1053.714^{\mathrm{d}}$ & {$[17]$} \\
anti-o-Cresol & $a n t i-o-\mathrm{C}_{6} \mathrm{H}_{4} \mathrm{CH}_{3} \mathrm{OH}$ & $533.53 \pm 0.58$ & {$[18]$} \\
$o$-Tolunitrile & $o-\mathrm{C}_{6} \mathrm{H}_{4} \mathrm{CH}_{3} \mathrm{CN}$ & $534.614^{\mathrm{e}}$ & {$[10]$} \\
& & $537.71 \pm 0.10$ & {$[11]$} \\
$o$-Toluidine & & $2002.3^{\mathrm{e}}$ & This work \\
\hline
\end{tabular}

${ }^{\mathrm{d}}$ Converted from $\mathrm{kJ} / \mathrm{mol}$ to $\mathrm{Cal} / \mathrm{mol}$, ${ }^{\mathrm{e}}$ converted from $\mathrm{cm}^{-1}$ to $\mathrm{Cal} / \mathrm{mol}$.

TABLE 6: Bond distances, angles, and dihedral angles of $o$-tolunitrile calculated on DFT B3LYP/6-311G (3d, p) level.

\begin{tabular}{|c|c|c|c|c|c|}
\hline \multicolumn{2}{|c|}{ Distances (pm) } & \multicolumn{2}{|c|}{ Angles $\left({ }^{\circ}\right)$} & \multicolumn{2}{|c|}{ Dihedral angles $\left({ }^{\circ}\right)$} \\
\hline $\mathrm{C} 1-\mathrm{C} 2$ & 140.04 & $\mathrm{C} 1-\mathrm{C} 2-\mathrm{C} 3$ & 120.0508 & & \\
\hline $\mathrm{C} 2-\mathrm{C} 3$ & 138.70 & $\mathrm{C} 2-\mathrm{C} 3-\mathrm{C} 4$ & 119.5363 & & \\
\hline $\mathrm{C} 3-\mathrm{C} 4$ & 139.12 & C3-C4-C5 & 120.2915 & & \\
\hline C4-C5 & 139.15 & C4-C5-C6 & 121.4869 & & \\
\hline C5-C6 & 139.39 & C5-C6-C1 & 117.5412 & & \\
\hline C1-C6 & 140.97 & C1-C6-C11 & 121.0341 & & \\
\hline $\mathrm{C} 2-\mathrm{H} 7$ & 108.29 & $\mathrm{C} 3-\mathrm{C} 2-\mathrm{H} 7$ & 120.7200 & & \\
\hline $\mathrm{C} 3-\mathrm{H} 8$ & 108.32 & $\mathrm{C} 4-\mathrm{C} 3-\mathrm{H} 8$ & 120.4898 & & \\
\hline C4-H9 & 108.39 & C5-C4-H9 & 119.6849 & & \\
\hline $\mathrm{C} 1-\mathrm{C} 15$ & 143.16 & $\mathrm{C} 2-\mathrm{C} 1-\mathrm{H} 15$ & 119.3137 & & \\
\hline C15-N16 & 116.77 & C6-C1-C15 & 119.8284 & & \\
\hline C6-C11 & 150.53 & C5-C6-C11 & 121.3573 & & \\
\hline C5-H10 & 108.44 & C6-C5-H10 & 118.8873 & & \\
\hline $\mathrm{C} 11-\mathrm{H} 12$ & 109.33 & C6-C11-H12 & 111.1996 & C1-C6-C11-H12 & 59.4310 \\
\hline $\mathrm{C} 11-\mathrm{H} 13$ & 109.04 & C6-C11-H13 & 110.9089 & C1-C6-C11-H13 & -179.9726 \\
\hline $\mathrm{C} 11-\mathrm{H} 14$ & 109.33 & C6-C11-H14 & 111.2012 & C1-C6-C11-H14 & -59.3797 \\
\hline
\end{tabular}

namely, $V_{3}$ and $\theta_{a}$ were allowed to vary and $I_{\alpha}$ was fixed to $3.2 \mathrm{u} \AA^{2}$ [4]. The "true" rotational and centrifugal distortion constants reported in [11] were used as fixed parameters in these fits. The results of the internal rotation analysis are presented in Table 4. A comparison of Fit 1 and Fit 2 reveals that parameters in Fit 2 are more accurately determined than that of Fit 1.

\section{DFT Calculation}

Quantum mechanical calculations were performed via the density functional theory (DFT) using Gaussian 03 package [21] implemented on Intel Core 2 machine. The calculations were performed to determine the structural parameters, rotational constants, centrifugal distortion constants andinternal rotation parameters $I_{\alpha}, \theta_{a}, \theta_{b}$, and barrier to internal rotation $\left(V_{3}\right)$ of the methyl top of $o$-tolunitrile. The moment of inertia of the methyl group $I_{\alpha}$ is obtained by assuming $\mathrm{C} 6-\mathrm{C} 11$ bond as fixed axis of rotation and $\theta_{a}$, $\theta_{b}$ were obtained from structural data. The geometry optimization is carried out using Becke three parameter hybrid functional and Lee, Yang, Parr nonlocal correlation functional (B3LYP) with relatively large basis set 6-311G (3d, p). The torsional potential curve of the methyl top is obtained by varying the dihedral angle of one methyl hydrogen from $0^{\circ}$ to $360^{\circ}$ relative to the molecular framework in steps of $10^{\circ}$ on the optimized geometry. The molecular drawing is done by using GaussView 3.0 software package [22]. The number and labeling of $o$-tolunitrile molecule is shown in Figure 1.

All torsional potential values were calculated with respect to the lowest energy configuration of $o$-tolunitrile. The calculated potential function is shown in Figure 2. The potential barrier $V_{3}$ calculated by DFT method is found to be $608.41 \mathrm{cal} / \mathrm{mol}$ which is comparable with the experimental value of $537.71 \mathrm{cal} / \mathrm{mol}$. The calculated values of rotational constants, centrifugal distortion constants, and internal rotation parameters obtained from DFT theoretical calculations have been compared with the corresponding experimental 
values and are shown in Table 4. The agreement is found to be highly satisfactory.

In Table 5 the potential barrier $V_{3}$ values of $o$-tolunitrile have been compared with other related molecules. Structural parameters of $o$-tolunitrile have been computed and are shown in Table 6.

\section{Conclusions}

The millimeterwave rotational spectra of $o$-tolunitrile have been measured in the frequency range $50.0-75.0 \mathrm{GHz}$ in the ground torsional state. Many high- $J\left(J_{\max }=30\right)$ rotational transitions with large A-E splitting have been assigned. The analysis of these splitting allows us to determine the internal rotation parameters more accurately. A comparison of the $V_{3}$ values of different toluene derivatives (Table 5) indicates that a heavier substituent such as a chlorine atom or methyl groups on the toluene ring leads to an enhancement of the torsional barrier values from the corresponding $V_{3}$ values obtained in cases where a lighter substituent such as a fluorine atom or a cyanogen group is attached to the ring. To support the experimental results, DFT calculations were carried out with DFT/B3LYP/6-311G (3d, p) basis set. The parameters obtained by DFT calculations were found to be in good agreement with those observed experimentally.

\section{Acknowledgments}

A. I. Jaman would like to thank Mr. A. K. Bhattacharya for his technical assistance during the course of this paper. P. R. Bangal gratefully acknowledges support from the DST, Government of India, Grant no. SR/FTP/CS-93/2005.

\section{References}

[1] H. D. Rudolph, H. Dreizler, A. Jaeschke, and P. Wendling, "Mikrowellenspektrum, hiderungspotential der internen rotation und dipolmoment des toluols," Zeitschrift für Naturforschung, vol. 22, pp. 940-944, 1967.

[2] J. Susskind, "Microwave spectrum, barrier to internal rotation, and torsion-vibration interaction in ortho-fluoro toluene," The Journal of Chemical Physics, vol. 53, no. 6, pp. 2492-2501, 1970.

[3] H. D. Rudolph and A. Trinkaus, "Mikrowellenspektrum, hinderungspotential der internen rotation und dipolmoment des meta-fluorotoluols," Zeitschrift für Naturforschung, vol. 23, pp. 68-76, 1968.

[4] H. D. Rudolph and H. Seiler, "Mikrowellenspektrum, hinderungspotential der internen rotation und dipolmoment des para-fluorotoluols," Zeitschrift für Naturforschung, vol. 20, pp. 1682-1686, 1965.

[5] S. Maiti, A. I. Jaman, and R. N. Nandi, "Microwave spectrum and barrier to internal rotation in 2,4-difluorotoluene," Journal of Molecular Spectroscopy, vol. 177, no. 1, pp. 29-33, 1996.

[6] K. Okuyama, N. Mikami, and M. Ito, "Internal rotation of the methyl group in the electronically excited state: $o$-and $m$ toluidine," Laser Chemistry, vol. 7, pp. 197-212, 1987.

[7] T. Aota, T. Ebata, and M. Ito, "Rotational isomers and internal rotation of the $\mathrm{CH} 3$ group in S0, S1, and ion of $o$-cresol," Journal of Physical Chemistry, vol. 93, no. 9, pp. 3519-3522, 1989.
[8] H. Mizuno, K. Okuyama, T. Ebata, and M. Ito, "Rotational isomers of $m$-cresol and internal rotation of the $\mathrm{CH} 3$ group in S0, S1, and the ion," Journal of Physical Chemistry, vol. 91, no. 22, pp. 5589-5593, 1987.

[9] M. Fujii, M. Yamauchi, K. Takazawa, and M. Ito, "Electronic spectra of $\mathrm{o}^{-}, \mathrm{m}$ - and p-tolunitrile-substituent effect on internal rotation of the methyl group," Spectrochimica Acta A, vol. 50, no. 8-9, pp. 1421-1433, 1994.

[10] A. I. Jaman, S. Maiti, and R. N. Nandi, "Microwave spectrum and barrier to internal rotation in ortho-tolunitrile," Journal of Molecular Spectroscopy, vol. 192, no. 1, pp. 148-151, 1998.

[11] N. Hansen, H. Mäder, and T. Bruhn, "A molecular beam Fourier transform microwave study of o-tolunitrile: ${ }^{14} \mathrm{~N}$ nuclear quadrupole coupling and methyl internal rotation effects," Molecular Physics, vol. 97, no. 4, pp. 587-595, 1999.

[12] W. Kasten, Ph.D. thesis, University of Kiel, 1985.

[13] A. Bauder and H. H. Günthard, "Internal rotation in acetaldehyde," Journal of Molecular Spectroscopy, vol. 60, no. 1-3, pp. 290-311, 1976.

[14] R. C. Woods, "A general program for the calculation of internal rotation splittings in microwave spectroscopy," Journal of Molecular Spectroscopy, vol. 21, no. 1-4, pp. 4-24, 1966.

[15] D. Gerhard, A. Hellweg, I. Merke et al., "Internal rotation and chlorine nuclear quadrupole coupling of $o$-chlorotoluene studied by microwave spectroscopy and ab initio calculations," Journal of Molecular Spectroscopy, vol. 220, no. 2, pp. 234-241, 2003.

[16] K. P. R. Nair, J. Demaison, G. Wlodarczak, and I. Merke, "Millimeterwave rotational spectrum and internal rotation in o-chlorotoluene," Journal of Molecular Spectroscopy, vol. 237, no. 2, pp. 137-142, 2006.

[17] H. D. Rudolph, K. Walzer, and I. Krutzik, "Microwave spectrum, barrier for methyl rotation, methyl conformation, and dipole moment of ortho-xylene," Journal of Molecular Spectroscopy, vol. 47, no. 2, pp. 314-339, 1973.

[18] A. Welzel, A. Hellweg, I. Merke, and W. Stahl, "Structural and torsional properties of o-cresol and o-cresol-OD as obtained from microwave spectroscopy and ab initio calculations," Journal of Molecular Spectroscopy, vol. 215, no. 1, pp. 58-65, 2002.

[19] D. R. Herschbach, "Calculation of energy levels for internal torsion and over-all rotation. III," The Journal of Chemical Physics, vol. 31, no. 1, pp. 91-108, 1959.

[20] J. K. G. Watson, in Vibrational Spectra and Structure, J. R. Durig, Ed., vol. 6, pp. 1-89, Marcel Dekker, New York, NY, USA, 1977.

[21] Gaussian 03, Revision-B.03, Gaussian, Pittsburgh, Pa, USA, 2003.

[22] GaussView3.0, Gaussian, Pittsburgh, Pa, USA. 

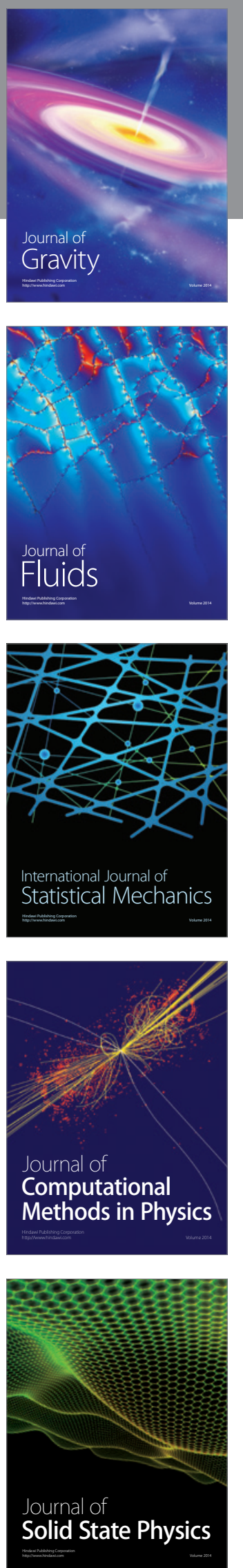

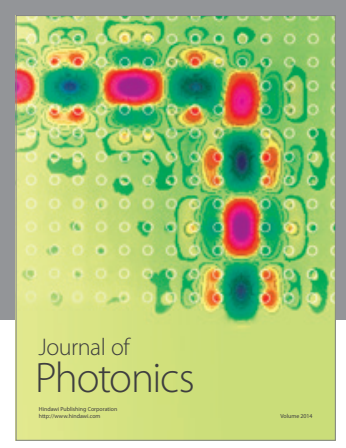

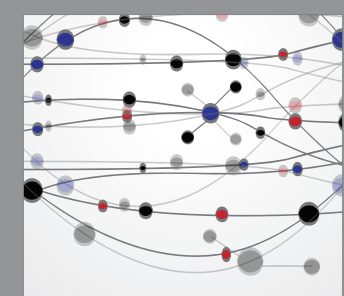

The Scientific World Journal
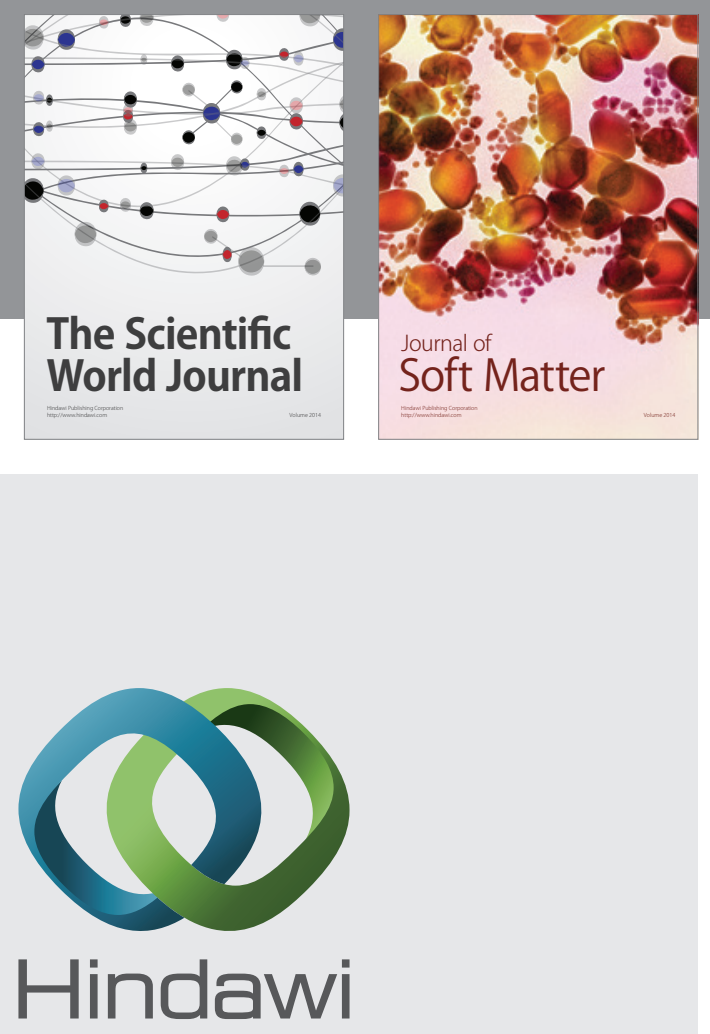

Submit your manuscripts at

http://www.hindawi.com
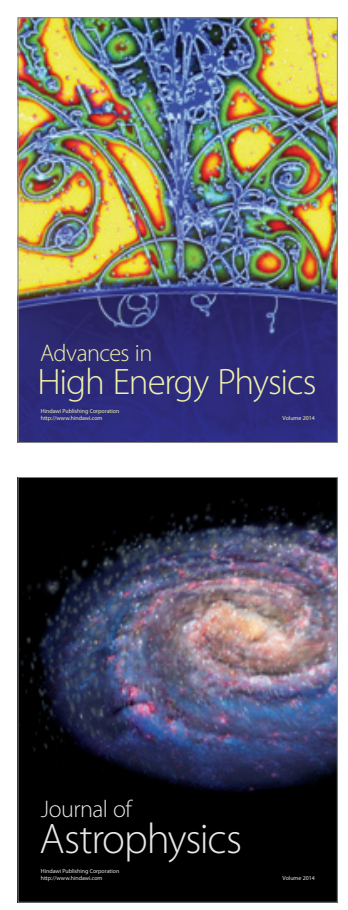
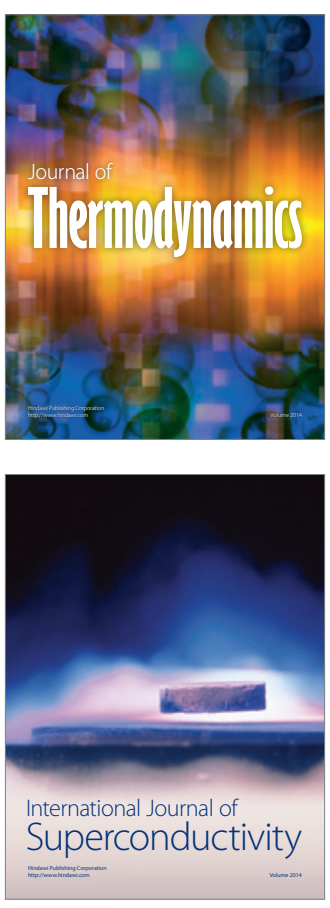
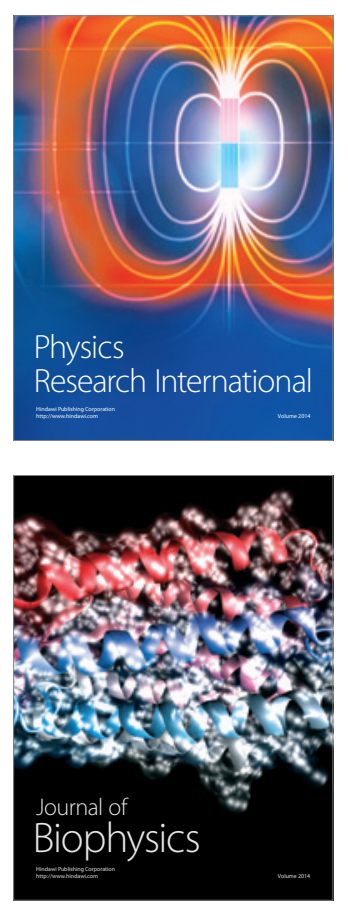
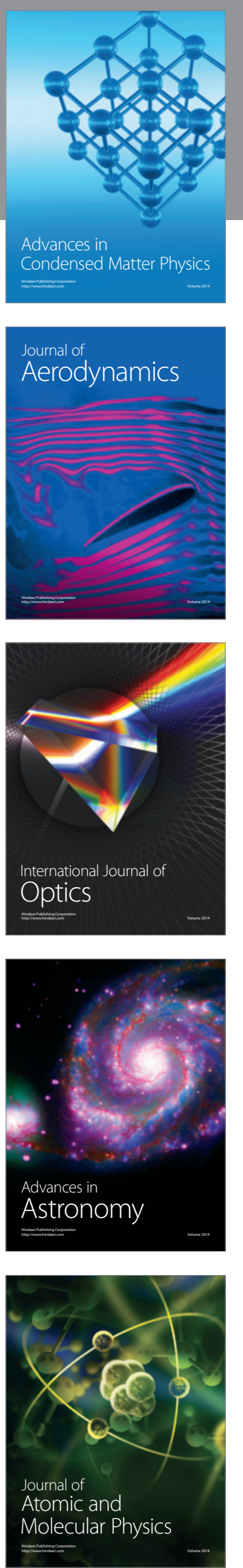